\title{
EFEITO DO POTÁSSIO NA FASE DE RUSTIFICAÇÃO DE MUDAS CLONAIS DE EUCALIPTO ${ }^{1}$
}

\author{
Flavio Siqueira D’Avila², Haroldo Nogueira de Paiva ${ }^{3}$, Helio Garcia Leite ${ }^{3}$, Nairam Félix de Barros ${ }^{3}$ e \\ Fernando Palha Leite ${ }^{4}$
}

\begin{abstract}
RESUMO - A aplicação de K está muitas vezes relacionada ao processo de rustificação, que tem por objetivo adaptar a muda às condições de plantio no campo. Neste sentido, este experimento foi conduzido visando avaliar o efeito do K na qualidade de mudas de três clones híbridos de Eucalyptus (E. grandis x E. urophylla). O experimento foi instalado no viveiro de mudas da empresa CENIBRA. As doses de potássio utilizadas foram: 0, 249, 747, 1494 e $2988 \mathrm{mg} \mathrm{L}^{-1}$. Aos 87 e 88 dias, foram avaliadas a altura da parte aérea, incremento em diâmetro de colo, relação altura da parte aérea/diâmetro de colo, concentração de K nas folhas e a relação N/K nas folhas. A aplicação de K na fase de rustificação aumentou, até determinada dose, o diâmetro de colo para os três clones avaliados, mas não se verificou influência do K na altura. A ausência de K resultou nos maiores valores da relação altura: diâmetro de colo para os três clones avaliados, ao passo que em mudas onde o K foi aplicado, esta relação apresentou valores menores e semelhantes. A aplicação de doses crescentes de $\mathrm{K}$ aumentou as concentrações foliares do nutriente. Todos os tratamentos apresentaram valores da relação $\mathrm{N} / \mathrm{K}$ dentro do considerado adequado, exceto na maior dose de K aplicada. Conclui-se que, durante a fase de rustificação, a adubação potássica é recomendável, sendo indicada a dose de $249 \mathrm{mg} \mathrm{L}^{-1}$ de $\mathrm{K}_{\text {por proporcionar }}$ os maiores valores em incremento de diâmetro de colo.
\end{abstract}

Palavras chave: Eucalyptus, miniestaquia e rustificação.

\section{EFFECT OF POTASSIUM ON THE HARDENING PHASE OF CLONAL SEEDLINGS OF EUCALYPT}

\begin{abstract}
The K is often related to the hardening process, in order to adapt the seedling to the field planting conditions. Thus, the objective of this experiment was evaluate the effect of $K$ on the quality of seedlings of three hybrids clones of Eucalyptus (E. grandis $x$ E. urophylla). The experiment was carried out in the seedling nursery of the CENIBRA enterprise. The doses of $K$ was: 0, 249, 747, 1494 and $2988 \mathrm{mg} \mathrm{L}^{-1}$. With 87 and 88 days, were avaluated: the shoot height, collar diameter increment, ratio shoot height / collar diameter, K concentration in leaves and N/K ratio in leaves. The K application in the hardening phase increased, up to a given dose, the collar diameter for the three clones evaluated, but the height was not influenced. The absence of K resulted in the greatest values of the ration height / collar diameter for the three clones evaluated, while on the seedlings that received $K$, the rate showed smaller and similar values. The application with increasing doses of $K$ resulted in greater leaf concentrations of this nutrient. All the treatment showed values of N/K ratio within what is considered appropriate, except for the greatest level of $K$. It can be concluded that, during the hardening phase, the potassic fertilization is recommended, and being indicated the dose of $249 \mathrm{mg} \mathrm{L}^{-1}$ of $\mathrm{K}$ because provides the best values of collar diameter increment.
\end{abstract}

Keywords: Eucalyptus, minicutting and hardening.

\footnotetext{
${ }^{1}$ Recebido em 30.06.2008 e aceito para publicação em 25.08.2010.

${ }^{2}$ Companhia Vale do Araguaia. E-mail: <flavio.davila@ufv.br>.

${ }^{3}$ Universidade Federal de Viçosa, UFV, Brasil. E-mail: <hnpaiva@ufv.br>.

${ }^{4}$ CENIBRA - Celulose Nipo-Brasileira S/A. E-mail: < fernando.leite@cenibra.com.br>.
} 


\section{INTRODUÇÃO}

Entre outros fatores de natureza silvicultural, a nutrição de mudas desponta como um dos principais responsáveis pela obtenção de maior produtividade e qualidade, além de maior economicidade no processo de sua produção (NEVES et al., 1990). A fertilização mineral afeta o desenvolvimento e qualidade de mudas de espécies florestais ao permitir melhor adaptação e consequente sobrevivência após o plantio no campo (CARNEIRO, 1995).

Dentro do processo de produção de mudas de eucalipto, a fase de rustificação trata de preparar a muda fisiologicamente para o plantio nas primeiras semanas que o sucedem. Nesse período, as mudas deverão resistir ao estresse provocado pelas atividades de plantio (falta de água, retirada dos tubetes, transporte). Antes da expedição, durante a rustificação normalmente se efetuam até três fertirrigações diárias, priorizando formulações com reduzida relação N/K, visto ser essa fase extremamente crítica e refletir diretamente na qualidade final das mudas (ALFENAS et al., 2004). Segundo Gomes e Couto (1984), para que a muda seja considerada apta para ser levada ao campo, ela deve ser sadia e ter grau de resistência que lhe permita sobreviver às condições adversas do meio.

Em mudas de eucalipto, aspecto ainda carente de informações vem a ser o efeito que a fertilização potássica pode proporcionar à resistência das mudas (BARROS et al., 1990). Plantas deficientes em potássio apresentam menor turgor, pequena expansão celular, maior potencial osmótico e abertura e fechamento dos estômatos de forma irregular (MALAVOLTA et al., 1997).

O potássio não faz parte de nenhum composto orgânico, não desempenhando função estrutural na planta. Esse macronutriente atua na ativação de aproximadamente 50 enzimas, destacando-se as sintetases, oxirredutases, desidrogenases, transferases, quinases e aldolases (MARSCHNER, 1995). O potássio é o principal íon presente no vacúolo das células relacionado ao ajustamento osmótico; logo, é importante na manutenção do turgor, principalmente sob condições de baixo potencial hídrico (BOURNE et al., 1988). Silveira e Malavolta (2000) destacaram a importância do K, relatando que plantas bem nutridas com esse nutriente são mais resistentes a secas e geadas, em razão da maior retenção de água.

Revista Árvore, Viçosa-MG, v.35, n.1, p.13-19, 2011
Com base no exposto, este trabalho teve por objetivo avaliar o efeito do potássio na qualidade e nutrição de mudas de três clones de Eucalyptus spp. durante a fase de rustificação.

\section{MATERIAIL E MÉTODOS}

Os ensaios foram conduzidos no viveiro de mudas da Celulose Nipo-Brasileira S. A. - CENIBRA, em Belo Oriente, MG. O município localiza-se na região do Vale do Rio Doce, com clima do tipo Cwa (subtropical, chuvoso e mesotérmico), segundo a classificação de Koeppen. A precipitação média anual é de 1.233 mm e temperatura média anual de $21^{\circ} \mathrm{C}$, com máxima média de $27^{\circ} \mathrm{C}$ e mínima média de $14^{\circ} \mathrm{C}$. O viveiro de mudas localiza-se na latitude $19^{\circ} 17^{\prime} 49^{\prime \prime}$ Sul, longitude $42^{\circ} 23^{\prime} 26^{\prime \prime}$ Oeste e altitude de 233 m (MAFIA et al., 2006).

Todos os procedimentos de análise química e pesagem de material vegetal foram realizados no laboratório de solos e análise de plantas da mesma empresa.

Foram utilizadas miniestacas oriundas de três clones de Eucalyptus grandis x Eucalyptus urophylla cultivados em minijardim clonal em leito de areia. Os clones utilizados foram 386, 1.213 e 3.837.

O estaqueamento foi realizado em substrato acondicionado em tubetes plásticos com dimensões de 12,5 cm de comprimento, 2,7 cm de diâmetro da abertura superior, 0,9 cm de diâmetro de abertura inferior e volume de $50 \mathrm{~cm}^{3}$, com seis estrias internas. Como suporte para os tubetes foram utilizadas bandejas com dimensões de 58,9 cm x 38,6 cm com capacidade para 176 mudas. O substrato utilizado foi vermiculita e casca de arroz carbonizada na proporção de 1:1, em volume, adubado de acordo com as indicações da Tabela 1.

As mudas iniciaram enraizamento em casa vegetação, aí permanecendo por 18 dias. Após esse período, foram transferidas para casa de sombra, onde permaneceram por mais seis dias. Na próxima etapa, o crescimento das mudas realizou-se em mesas rolantes recebendo a fertirrigação pelo sistema de irrigação na subsuperficie e aspersão por 44 dias (Tabela 2). Por fim, permaneceram por mais 20 dias no estaleiro, onde os tratamentos com $\mathrm{K}$ via água foram realizados.

Até o $68^{\circ}$ dia, antes de as mudas chegarem ao estaleiro todas receberam as mesmas adubações. A partir dessa data, aplicaram-se as cinco doses de K até o $88^{\circ}$ dia, quando o experimento foi finalizado. 
Tabela 1 - Concentração de nutrientes e fontes utilizadas na adubação de substrato de enraizamento.

Table 1 - Nutrient concentrations and sources used on the fertilization of rooting substrate.

\begin{tabular}{|c|c|}
\hline Nutrientes & $\begin{array}{l}\text { Substrato de enraizamento } \\
\text { mg de nutriente por litro }\end{array}$ \\
\hline $\begin{array}{c}\mathbf{P} \\
\text { (super fosfato simples) }\end{array}$ & 698,0 \\
\hline $\begin{array}{c}\mathbf{N} \\
\text { (sulfato de amônio) }\end{array}$ & 146,0 \\
\hline $\begin{array}{c}\mathbf{K} \\
\text { (cloreto de potássio) }\end{array}$ & 99,0 \\
\hline $\mathrm{S}$ & 176,0 \\
\hline $\begin{array}{c}\text { B } \\
\text { (ácido bórico) }\end{array}$ & 4,9 \\
\hline $\begin{array}{c}\mathbf{C} \mathbf{u} \\
\text { (sulfato de cobre) }\end{array}$ & 5,6 \\
\hline $\begin{array}{c}\text { Mn } \\
\text { (sulfato de manganês) }\end{array}$ & 5,1 \\
\hline $\begin{array}{c}\text { Zn } \\
\text { (sulfato de zinco) }\end{array}$ & 6,0 \\
\hline
\end{tabular}

Tabela 2 - Concentração de nutrientes e fontes utilizadas na fertirrigação de mudas em casa de sombra e irrigação na subsuperficie.

Table 2 - Nutrient concentrations and sources used in the fertirrigation of seedling in the shed and benches.

\begin{tabular}{|c|c|c|}
\hline \multirow[t]{2}{*}{ Nutrientes } & Casa de sombra & Subirrigação \\
\hline & \multicolumn{2}{|c|}{ mg de nutriente por litro } \\
\hline $\begin{array}{c}\mathbf{N} \\
\text { (nitrato de cálcio) }\end{array}$ & 56 & 146 \\
\hline $\begin{array}{c}\mathbf{P} \\
\text { (ácido fosfórico) }\end{array}$ & 42 & 108 \\
\hline $\begin{array}{c}\mathbf{K} \\
\text { (cloreto de potássio) }\end{array}$ & 87 & 225 \\
\hline $\mathbf{C a}$ & 49 & 127 \\
\hline $\begin{array}{c}\text { Mg } \\
\text { (sulfato de magnésio) }\end{array}$ & 61 & 159 \\
\hline $\mathrm{S}$ & 20 & 53 \\
\hline $\begin{array}{c}\mathbf{B} \\
\text { (ácido bórico) }\end{array}$ & 0,80 & 2,1 \\
\hline $\begin{array}{c}\mathbf{C} \mathbf{u} \\
\text { (sulfato de cobre) }\end{array}$ & 0,19 & 0,5 \\
\hline $\begin{array}{c}\mathbf{F e} \\
\text { (sulfato ferroso) }\end{array}$ & 3,50 & 9,0 \\
\hline $\begin{array}{c}\text { Mn } \\
\text { (sulfato de manganês) }\end{array}$ & 0,80 & 2,0 \\
\hline $\begin{array}{c}\text { Mo } \\
\text { (molibdato de sódio) }\end{array}$ & 0,04 & 0,11 \\
\hline $\begin{array}{c}\text { Zn } \\
\text { (sulfato de zinco) }\end{array}$ & 0,11 & 0,28 \\
\hline
\end{tabular}

Os tratamentos consistiram em quatro doses de K na forma de cloreto de potássio, mais uma testemunha, que recebeu apenas água. O delineamento estatístico adotado foi o inteiramente casualizado. Foram quatro repetições por tratamento, e cada repetição continha 16 mudas. As doses utilizadas foram: 0, 249, 747, 1.494 e $2.988 \mathrm{mg} \mathrm{L}^{-1}$ de $\mathrm{K}$, identificadas como K1, K2, K3, K4 e K5, respectivamente.

Ao final do dia, quando as irrigações via aspersão eram suspensas, realizava-se a aplicação do KCl com o auxílio de regador, sendo aplicados $4 \mathrm{~L}$ da solução nutritiva por tratamento.

As características morfológicas avaliadas aos 87 e 88 dias foram altura da parte aérea, incremento em diâmetro de colo e a relação altura da parte aérea/diâmetro de colo. Os instrumentos utilizados nas determinações foram: régua e paquímetro. Considerou-se como altura da parte aérea o comprimento entre a base do caule em contato com a superfície do substrato e a base do par de folhas mais jovem.

As medições do diâmetro de colo foram realizadas em duas ocasiões: no $68^{\circ}$ e $69^{\circ}$ dia e no $87^{\circ}$ e $88^{\circ}$ dia, com a finalidade de avaliar a diferença de crescimento das mudas (incremento) em um período de 20 dias. No $88^{\circ}$ dia, realizou-se a coleta do material vegetal para a pesagem e análise química das folhas. Para a análise química foram colhidos os penúltimos e os antepenúltimos pares de folhas recém-maduras. A análise do tecido vegetal foi feita por digestão nitroperclórica e as leituras, através da fotometria de chama.

Os dados de altura e incremento em diâmetro de colo foram submetidos à análise de variância e, quando foi possível, à análise de regressão, com os softwares Estatística 8.0 (STATSOFT INC., 2008) e Curve Expert (CURVE EXPERT, 2007). Utilizou-se a análise estatística descritiva dos dados da relação altura da parte aérea/ diâmetro de colo, devido à presença de heterocedasticidade e à não observância da distribuição normal dos erros para esta variável. Para a concentração de K foliar foi realizada análise de regressão, sendo ajustado uma regressão exponencial.

\section{RESULTADOS E DISCUSSÕES}

A dose de $\mathrm{K}$ influenciou apenas no incremento em diâmetro de colo, não havendo interação entre clone e dose de K (Tabela 3). Desse modo, foram utilizados os dados dos três clones conjuntamente, para a análise de regressão do incremento em diâmetro de colo.

Revista Árvore, Viçosa-MG, v.35, n.1, p.13-19, 2011 
Tabela 3 - Análise de variância da altura da parte aérea e incremento em diâmetro de colo de mudas aos 88 dias de idade, em função de doses de $\mathrm{K}$ nos três clones de eucalipto.

Table 3 - Variance analyses of height of the aerial part and collar diameter increment of the seedlings at 88 days related to rates of $K$ for the three clones of eucalypt.

\begin{tabular}{lccc}
\hline \multirow{2}{*}{ FV } & GL & \multicolumn{2}{c}{ Quadrado médio } \\
\cline { 2 - 4 } & & Altura & $\begin{array}{c}\text { Incremento em } \\
\text { diâmetro de colo }\end{array}$ \\
\hline Clone & 2 & $82,24 *$ & $0,039585 *$ \\
Dose (K) & 4 & $6,33 \mathbf{n s}$ & $0,137221 *$ \\
Clone $\mathbf{x}$ K & 8 & 8,35 ns & 0,002046 ns \\
Resíduo & 45 & 4,31 & 0,011408 \\
Total & 59 & & \\
\hline
\end{tabular}

* Teste F significativo em nível de $\mathrm{P}<0,05$.

Provavelmente, a não influência das doses de K na altura foi devida ao fato de as mudas já se encontrarem na fase de rustificação. Nessa fase, a muda em tubete já passou pela fase de crescimento rápido, pois o tamanho da embalagem e, consequentemente, a quantidade de substrato e outros nutrientes são limitantes. Silva et al. (2004) ressaltaram que a fase de rustificação não tem por objetivo o desenvolvimento da muda e, sim, a aclimatação para as condições de campo. No entanto, durante a fase de crescimento, período que antecede a rustificação, o nutriente se mostra importante para a característica altura, como observado em estudo realizado por Brandi et al. (1982) avaliando efeito de macronutrientes no crescimento inicial de Eucalyptus spp, em que ocorreu grande redução nos incrementos em altura de E. paniculata na ausência de $\mathrm{K}$.

Segundo Gomes et al. (2003), apesar de ainda existirem controvérsias sobre o tamanho de mudas de eucalipto, considera-se que elas poderiam estar prontas para serem plantadas com altura variando de 20 a $35 \mathrm{~cm}$. Dessa forma, independentemente do tratamento, a altura atingida por todos os clones atendeu ao padrão estabelecido para plantio (Tabela 4).

O incremento em diâmetro de colo em resposta ao K foi mais bem representado por equação quadrática, com elevação até a dose de $1.655 \mathrm{mg} \mathrm{L}^{-1}$ de K, decrescendo a partir daí (Figura 1). Para qualquer dose de potássio aplicada, os incrementos em diâmetro de colo foram maiores do que na ausência do nutriente (Figura 1). Desse modo, a aplicação do potássio até determinada dose mostrou resultado positivo para essa característica, visto um maior diâmetro de colo ser desejável das mudas
Tabela 4-Alturas de mudas de eucalipto aos 88 dias de idade, em função das doses de K nos clones 386, 1213 e 3837, durante a fase de rustificação.

Table 4-Height of seedlings of eucalypt at 88 days related to rates of $K$ for the clones 386, 1213 and 3837 during the hardening phase.

\begin{tabular}{cccc}
\hline Tratamento & \multicolumn{3}{c}{ Altura média $(\mathrm{cm})$} \\
\cline { 2 - 4 } & Clone 386 & Clone 1213 & Clone 3837 \\
\hline K1 & 31 & 30 & 28 \\
K2 & 33 & 28 & 30 \\
K3 & 32 & 31 & 27 \\
K4 & 34 & 31 & 29 \\
K5 & 33 & 28 & 31 \\
\hline
\end{tabular}

Obs.: K1 (0 mg L-1); K2 (249 mg L-1); K3 (747 mg L-1); K4 (1.494 $\left.\mathrm{mg} \mathrm{L}^{-1}\right)$ e $\mathrm{K} 5$ (2.988 $\left.\mathrm{mg} \mathrm{L}^{-1}\right)$.

durante a fase de rustificação. Segundo Gomes e Paiva (2004), as mudas devem ter maior diâmetro de colo para melhor equilíbrio do crescimento da parte aérea. Esse parâmetro é reconhecido como um dos melhores, senão o melhor indicador do padrão de qualidade de mudas (MOREIRA e MOREIRA, 1996) e, normalmente, é o mais observado para indicar a capacidade de sobrevivência da muda no campo (DANIEL et al., 1997). Entre as doses de $\mathrm{K}$ aplicadas, a de $249 \mathrm{mg} \mathrm{L}^{-1}$ seria a mais indicada, visto que todos os tratamentos que receberam $\mathrm{K}$ foram estatisticamente iguais, optando-se, portanto, pela menor dose (Tabela 5).

$$
\boldsymbol{Y}=(0,029502)+(0,0000288) * \mathrm{x}+(-0,0000000087) * \mathrm{x}^{2} \quad \mathrm{R}^{2}=0,33
$$

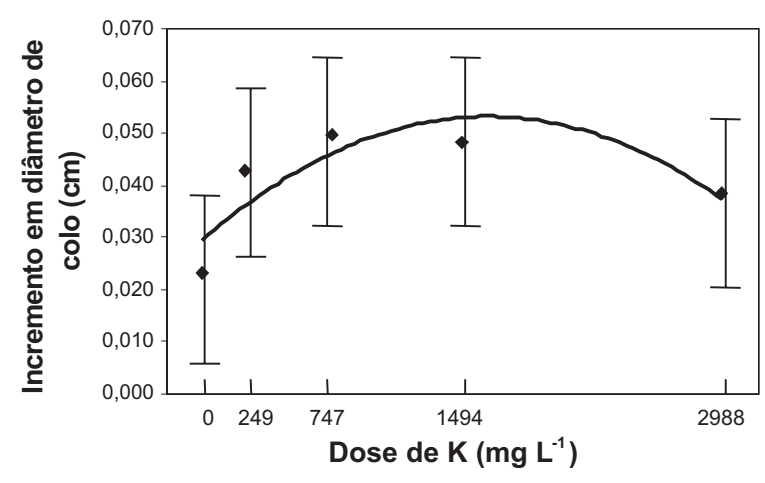

Figura 1 - Incremento em diâmetro de colo em função de doses de $\mathrm{K}$ avaliado durante 20 dias, na rustificação (dados dos três clones). Os pontos correspondem às médias e a linha ao modelo ajustado.

Figure 1 - Increment of the collar diameter in relation to the rates of K evaluated during 20 days in the hardening phase (data from the three clones). Bars represent the confidence interval, the dots correspond to the means and the lines the adjusted models. 
Tabela 5 - Médias inicial e final e incremento em diâmetro de colo, em função das doses de K.

Table 5 - Initial and final average and collar diameter increment related to the rates of $K$.

\begin{tabular}{ccccc}
\hline $\begin{array}{c}\text { Dose de } \mathrm{K} \\
\left(\mathrm{mg} \mathrm{L}^{-1}\right)\end{array}$ & $\begin{array}{c}\text { Medição } \\
\text { inicial }\end{array}$ & $\begin{array}{c}\text { Medição } \\
\text { final }\end{array}$ & $\begin{array}{c}\text { Incremento em } \\
\text { diâmetro de colo }\end{array}$ \\
\hline $\mathbf{0}$ & 3,10 & 3,30 & $0,20 \mathrm{a}$ & $\mathrm{a}$ \\
$\mathbf{2 4 9}$ & 2,95 & 3,40 & $0,45 \mathrm{~b}$ & $\mathrm{~b}$ \\
$\mathbf{7 4 7}$ & 3,00 & 3,55 & $0,55 \mathrm{~b}$ & $\mathrm{~b}$ \\
$\mathbf{1 4 9 4}$ & 3,05 & 3,55 & $0,50 \mathrm{~b}$ & $\mathrm{~b}$ \\
$\mathbf{2 9 8 8}$ & 3,10 & 3,50 & $0,40 \mathrm{~b}$ & $\mathrm{~b}$ \\
\hline
\end{tabular}

Médias seguidas pela mesma letra não diferem pelo teste de Tukey a 5\%.

Segundo Silva (1998), tão importante quanto as características altura da parte aérea e diâmetro de colo isoladamente é a relação entre eles. No ensaio deste trabalho, a ausência de potássio proporcionou os maiores valores da relação altura da parte aérea/diâmetro de colo nos três clones, ao passo que em mudas que receberam $\mathrm{K}$ a relação apresentou valores menores e semelhantes (Tabela 6).

Em trabalhos de pesquisa com espécies florestais, constatou-se que mudas com maior altura e maior diâmetro do colo apresentaram maior potencial de crescimento inicial no campo, entretanto a RHDC, quando correlacionada com a sobrevivência, tem apresentado resultados contraditórios (GOMES e PAIVA, 2004). Quanto menor for o seu valor, maior será a capacidade de as mudas sobreviverem e se estabelecerem na aérea de plantio definitivo (GOMES e PAIVA, 2004). Desse modo, verificou-se que a aplicação apenas de água durante a fase de rustificação - prática normalmente utilizada - foi menos eficiente quando comparada com os tratamentos que receberam $\mathrm{K}$, para obtenção de menor RHDC.

Tabela 6 - Relação altura:diâmetro de colo aos 88 dias de idade, em função das doses de K nos clones 386, 1213 e 3837, durante a fase de rustificação.

Table 6 - Rate height / collar diameter at 88 days related to the rates of $K$ for the clones 386, 1213 and 3837 during the hardening phase.

\begin{tabular}{cccc}
\hline Tratamento & \multicolumn{3}{c}{ Relação altura:diâmetro de colo } \\
\cline { 2 - 4 } & Clone 386 & Clone 1213 & Clone 3837 \\
\hline K1 & 92 & 96 & 90 \\
K2 & 83 & 83 & 81 \\
K3 & 83 & 82 & 79 \\
K4 & 84 & 83 & 78 \\
K5 & 86 & 85 & 82 \\
\hline
\end{tabular}

No ensaio deste estudo não se observou, visualmente, diferenciação no aspecto das plantas (tecido tenro ou “endurecido") entre os tratamentos. No entanto, o aparecimento de folhas avermelhadas nas mudas que receberam potássio iniciou-se próximo aos 20 dias após aplicação. Já Carneiro (1995) verificou que a presença desse nutriente em níveis crescentes aumentou a rustificação das mudas de espécies florestais e a consequente resistência às condições adversas do meio. Gonçalves (1995) verificou também efeito positivo do nutriente, recomendando adubação contendo apenas potássio no início da fase de rustificação, por facilitar o engrossamento do caule.

Doses crescentes de K resultaram em maiores concentrações foliares do nutriente, segundo o modelo ajustado para tal comportamento (Figura 2). Gonçalves (1995) relatou que a fertilização potássica melhora o metabolismo do K nas mudas, fazendo que elas sejam, fisiologicamente, mais capazes de regular suas perdas de água durante a fase de rustificação. Segundo Silveira e Malavolta (2000), plantas bem nutridas em K são mais resistentes a secas e geadas, em razão da maior retenção de água em seus tecidos.

As concentrações foliares de $\mathrm{K}$ em todos os tratamentos ficaram acima de $20 \mathrm{~g} \mathrm{~kg}^{-1}$ (Figura 2), valor esse sugerido como adequado por Silveira et al. (2001)

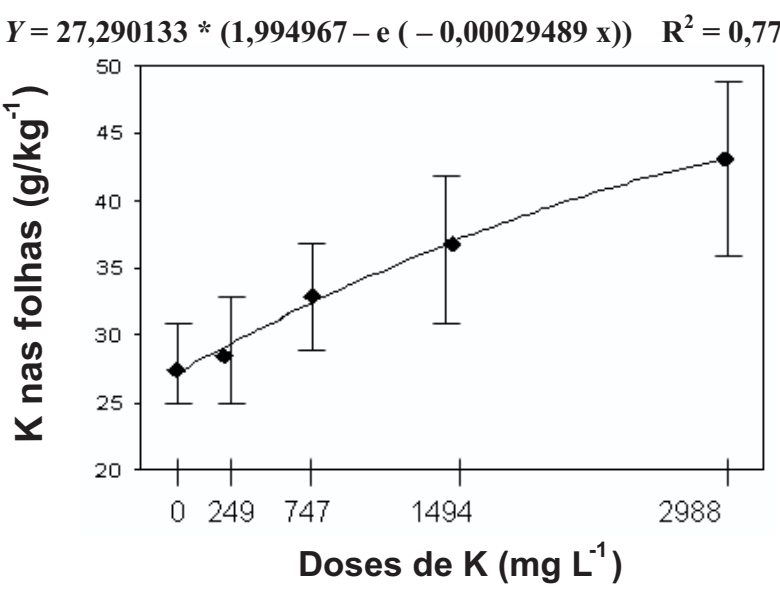

Figura 2 - Concentração foliar de K em função de doses de $\mathrm{K}$ aos 88 dias (dados dos três clones). As barras representam o intervalo de confiança, os pontos correspondem às médias e a linha ao modelo ajustado.

Figure 2 - Foliar concentration of $K$ related to the rates of $K$ at 88 days (data from the three clones). Bars represent the confidence interval, the dots correspond to the means, and the lines the adjusted models.

Revista Árvore, Viçosa-MG, v.35, n.1, p.13-19, 2011 
para mudas de E. grandis dos 80 aos 100 dias de idade. Até mesmo o tratamento que não recebeu adubação potássica durante essa fase apresentou valores acima do sugerido. Isso está relacionado às quantidades fornecidas de K durante a fase anterior à rustificação.

No referido ensaio foram encontrados os seguintes valores da relação N/K nas folhas dos tratamentos K1, K2, K3, K4 e K5, respectivamente 0,8; 0,8; 0,7; 0,6; e 0,5 no clone $386 ; 0,8 ; 0,8 ; 0,7 ; 0,6$; e 0,5 no clone 1213; e 0,8; 0,7; 0,6; 0,6; e 0,5 no clone 3837. Para Silveira et al. (2001), a relação N/K adequada nas folhas na fase inicial de crescimento está na faixa de 1,4 a 2,0, enquanto na fase de rustificação, entre 0,6 e 1,0. Desse modo, percebeu-se que em todos os clones, independentemente do tratamento, os valores estiveram dentro da faixa considerada adequada, à exceção do tratamento K5 (2.988 $\left.\mathrm{mg} / \mathrm{L}^{-1}\right)$.

\section{CONCLUSÕES}

Durante a fase de rustificação, a adubação potássica é recomendável, sendo a dose de $249 \mathrm{mg}$ $\mathrm{L}^{-1}$ de $\mathrm{K}$ indicada por proporcionar os maiores incrementos de diâmetro de colo.

\section{REFERÊNCIAS}

ALFEnAS, A. C. et al. Clonagem e doenças do eucalipto.Viçosa, MG: Universidade Federal de Viçosa, 2004. 442p.

BARROS, N. F.; NOVAIS, R. F.; NEVES, J. C. L. Fertilização e correção do solo para o plantio de eucalipto. In: BARROS, N. F.; NOVAIS, R. F., (Eds.) Relação solo-eucalipto. Viçosa, MG: Folha de Viçosa, 1990. p.127-186.

BOURNE, G. H.; JEON, K. W.; FRIEDLONGER, M. Potassium estimation uptake and its role in the physiology and metabolismo flowering plants. International Review of Cytology, v.110, p.205-254, 1988.

BRANDI, R. M. et al. Nutrição e dinâmica de elementos minerais em plantios de eucalipto no estado de Minas Gerais. Relatório anual apresentado pela coordenação do contrato IBDF/UFV/SIF ao Instituto Brasileiro de Desenvolvimento Florestal. Viçosa, MG: Universidade Federal de Viçosa, 1982.

Revista Árvore, Viçosa-MG, v.35, n.1, p.13-19, 2011
CARneiro, J. G. A. Produção e controle de qualidade de mudas florestais. Curitiba: UFPR/FUPEF, 1995. 451p.

CURVE EXPERT. A curve fitting system for Windows. Disponível em: <http:// curveexpert.webhop.biz/>. Acesso em: 01 dez. 2007.

DANIEL, O. et al. Aplicação de fósforo em mudas de Acacia mangium WILLD. Revista Árvore, v.21, n.2, p.163-168, 1997.

GOMES, J. M.; COUTO, L. Produção de mudas de Eucalyptus spp. no estado de Minas Gerais Evoluções e tendências. In: SIMPÓSIO INTERNACIONAL: MÉTODOS DE PRODUÇÃO E CONTROLE DE QUALIDADE DE SEMENTES E MUDAS FLORESTAIS. Anais...Curitiba: UFP/ FUPEF, 1984. p.440-458.

GOMES, J. M. et al. Crescimento de mudas de Eucalyptus grandis em diferentes tamanhos de tubetes e fertilização N-P-K. Revista Árvore, v.27, n.2, p.113-127, 2003.

GOMES, J. M.; PAIVA, H. P. Viveiros

florestais (propagação sexuada). Viçosa, MG: Universidade Federal de Viçosa, 2004. 116p. (Caderno Didático, 72).

GONÇALVES, J. L. M. Recomendações de adubação para Eucalyptus, Pinus e Espécies Típicas da Mata Atlântica. Piracicaba: Universidade de São Paulo, Escola Superior de Agricultura “Luiz de Queiroz”, 1995. 23p. (Documentos Florestais, n.15)

MAFIA, R. G. et al. Variáveis climáticas associadas à incidência de mofo-cinzento em eucalipto. Fitopatologia Brasileira, v.31, n.2, mar./abr., p.152-157, 2006.

MALAVOLTA, E.; VITTI, G. C.; OLIVEIRA, S. A. Avaliação do estado nutricional das plantas: princípios e aplicações. 2.ed. Piracicaba: Associação Brasileira para Pesquisa do Potássio e do Fosfato, 1997. 319p.

MARSCHNER, H. Mineral nutrition of higher plants. London: Academic Press, 1995. 889p. 
MOREIRA, F. M. S.; MOREIRA, F. W. Característica de germinação de 64 espécies de leguminosas florestais nativas da Amazônia, em condições de viveiro. Acta Amazônica, v.26, n.1/2, p.3-16, 1996.

NEVES, J. C. L.; GOMES, J. M.; NOVAIS, R. F. Fertilização mineral de mudas de eucalipto. In: BARROS, N. F.; NOVAIS, R. F. Relação solo-eucalipto. Viçosa, MG: Folha de Viçosa, 1990. p.100-124.

SILVA, M. R. Caracterização morfológica, fisiológica e nutricional de mudas de Eucalyptus grandis (Hill ex. Maiden) submetidas a diferentes níveis de estresse hídrico durante a fase de rustificação. 1998. 105f. Dissertação (Mestrado em Engenharia Florestal) - Setor de Ciências Agrárias, Universidade Federal do Paraná, Curitiba, 1998.
SILVA, M. R.; KLAR, A. E.; PASSOS, J. R. Efeitos do manejo hídrico e da aplicação de potássio nas características morfofisiológicas de mudas de Eucalyptus grandis W. (Hill ex. Maiden). Irriga, v.9, n.1, p.31-40, 2004.

SILVEIRA, R. L. V. A.; MALAVOLTA, E. Nutrição e adubação potássica em Eucalyptus. Piracicaba: 2000. 12p. (Informações Agronômicas, 91).

SILVEIRA, R. L. V. A. et al. Seja o doutor do seu eucalipto. Piracicaba: 2001. 32p.

(Informações Agronômicas, 93).

STATSOFT INC. Statistica data analysis system version 8.0. Tulsa: Statsoft Inc., 2008. 
